\title{
Vacancy-induced splitting of the Dirac nodal point in graphene
}

\author{
W. Zhu, ${ }^{1}$ W. Li, ${ }^{1, *}$ Q. W. Shi, ${ }^{1}$ X. R. Wang, ${ }^{2,3, \dagger}$ X. P. Wang, ${ }^{1}$ J. L. Yang, ${ }^{1}$ and J. G. Hou ${ }^{1}$ \\ ${ }^{1}$ Hefei National Laboratory for Physical Sciences at Microscale, University of Science and Technology of China, Hefei 230026, China \\ ${ }^{2}$ Department of Physics, The Hong Kong University of Science and Technology, Clear Water Bay, Kowloon, Hong Kong \\ ${ }^{3}$ School of Physics, Wuhan University, Wuhan, People's Republic of China
}

(Received 7 February 2012; published 27 February 2012)

\begin{abstract}
We investigate the vacancy effects on quasiparticle band structure of graphene near the Dirac point. It is found that each Dirac nodal point splits into two new nodal points due to the coherent multiple scattering among vacancies. The energy split between the two nodal points is proportional to the square root of vacancy concentration. In addition, an extra dispersionless band is developed at zero energy. Our theory offers an excellent explanation to the recent experiments.
\end{abstract}

DOI: 10.1103/PhysRevB.85.073407

PACS number(s): 81.05.ue, 71.23.-k, 71.55.-i

Electronic properties of materials are often determined by their low-energy excitations. The low-energy excitations of pristine grapheme form the Dirac nodal structures centered at two inequivalent corners $K$ and $K^{\prime}$ of the first Brillouin zone with linear energy dispersion. ${ }^{1,2}$ The common belief is that the Dirac nodal structure is robust against the shortranged potential scattering because long electron wavelength near the Dirac nodal point would not be able to "see such scatters." 3 However, recent progress in the classical wave physics demonstrates that local resonant structures can dramatically modify waves whose wavelengths are several orders of magnitude larger than the structure sizes. ${ }^{4-6}$ Vacancies as well as various chemical adsorbates in graphene can create resonant states in the vicinity of the Dirac point. Thus, similar to the classical wave, one expects that electronic structures and transport properties of graphene near the Dirac nodal point can be dramatically modified by vacancies. Indeed, the angleresolved photoemission spectroscopy (ARPES) indicates the opening of a tunable band gap near the Dirac point and the formation of a dispersionless band in hydrogenated quasi-freestanding graphene. ${ }^{7,8}$ Another study of hydrogenated graphene on $\mathrm{SiC}$ showed signals of a metal-to-insulator transition (MIT) due to the electron localization. ${ }^{9}$ Away from the charge neutrality point, the transport measurements demonstrated a sublinear carrier dependence of the conductivity. ${ }^{10}$ Within the Boltzmann transport framework and the assumption of existence of the Dirac nodal structure, theoretical prediction agrees with the experimental observation. ${ }^{11}$ However, it fails to explain the transport behavior near the nodal point, showing the breakdown of Boltzmann transport theory there. ${ }^{12,13}$ In fact, both experiments ${ }^{7,8}$ and numerical simulations ${ }^{14,15}$ suggest that the Dirac nodal structure is significantly changed by the resonant scattering. Therefore, the discrepancy between the theoretical prediction and transport measurements may arise from the change of quasiparticle behavior near the nodal point, and a deep understanding of the resonant scattering effects is needed.

In this Brief Report, we study the effects of the vacancy resonant scattering on Dirac nodal structure in graphene. The quasiparticle dispersion relation is extracted from the spectral function $A(\mathbf{k}, E)$, which can be calculated by extending the well-developed Lanczos approach. ${ }^{16}$ In contrast to the previous theoretical studies of the spectral function by the average $T$-matrix approximation (ATA) $)^{17-19}$ or the selfconsistent $T$-matrix approximation (SCTA), ${ }^{17,20}$ our method is more general and nonperturbative, including all the coherent multiple scattering contributions. We found that, instead of only one peak in the spectral function for a given momentum in the case of a weak short-range scattering, ${ }^{16}$ the resonant scattering yields multipeaks in the spectral function. This leads to a complete change of quasiparticle band structure. Each Dirac nodal point splits into two new nodal points and the energy split between the two nodal points is proportional to the square root of vacancy concentration. In addition, an extra dispersionless band is developed at zero energy. Our findings provide an accurate low-energy excitation spectrum near the charge neutrality point of graphene with vacancies that is useful for the Dirac nodal physics. They suggest also that the Boltzmann theory could work well near the Dirac point if the modified energy dispersion is taken into account.

$\pi$ electrons of pristine graphene can be modeled by a tight-binding Hamiltonian on a honeycomb lattice of two sites per unit cell, $H_{0}=t \sum_{\langle i j\rangle}|i\rangle\langle j|+$ H.c., where $t$ is the hopping energy between the nearest neighboring atoms. We consider vacancy disorder potential $V$ in this work because it gives rise to the resonant scattering, which may also arise from hydrogen or fluorine adsorbates. ${ }^{21}$ Vacancies are introduced by randomly removing lattice sites with probability $n_{\text {imp }}$ (vacancy concentration). To avoid any ambiguity arising from the imbalance of the vacancies in two sublattices, we keep the equal number of vacancies on the two sublattices in our calculations. ${ }^{22}$ In the presence of a disorder potential $V$, the single electron properties can be obtained from the ensemble-averaged Green function $G(\mathbf{k} \pm, E)=$ $\overline{\left\langle\mathbf{k} \pm\left|\frac{1}{E+i \eta-H_{0}-V}\right| \mathbf{k} \pm\right\rangle}$, where $|\mathbf{k} \pm\rangle$ is eigenstate of $H_{0}$ and $+(-)$ denotes the conduction (valence) band, respectively. This Green function can be obtained numerically by using the Lanczos recursive method. ${ }^{15,16,23-25}$ To numerically obtain an exact ensemble-averaged Green's function near the Dirac point, a large lattice containing millions sites $(4800 \times 4800)$ is used. The large samples guarantee that the calculated Green's function is free from the finite size errors. In the following calculations, the broadening parameter is set to be $\eta=$ $0.001 t .^{16}$ 

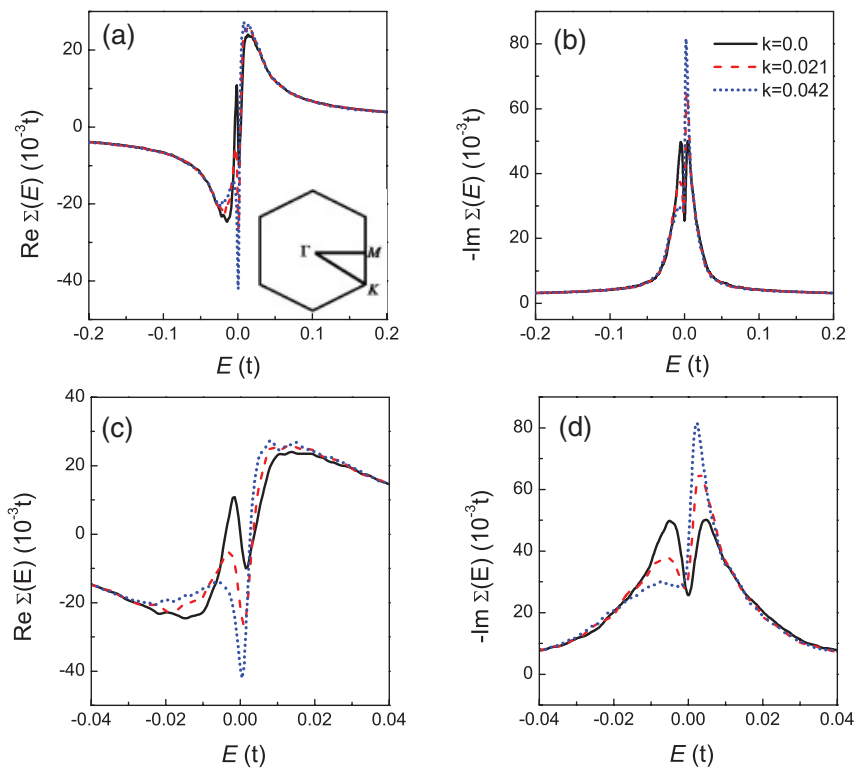

FIG. 1. (Color online) Real (a) and imaginary (b) parts of selfenergy function $\Sigma$ as a function of energy $E$ for various wave vectors in conduction band: $k=0$ (black solid line), 0.021 (red dashed line), and 0.042 (blue dotted line) (in unit of $1 / a$ ). The wave vector varies from the $K$ point to $M$ point in the Brillouin zone [Inset of (a)]. (c) and (d) are the enlarged views of (a) and (b) near the Dirac point $(E=0)$. The vacancy concentration is $n_{\text {imp }}=0.1 \%$.

Self-energy function $\Sigma$ is defined by the Dyson's equation as $G(\mathbf{k}, E)=G_{0}(\mathbf{k}, E)+G_{0}(\mathbf{k}, E) \Sigma(\mathbf{k}, E) G(\mathbf{k}, E)$. Thus, one has $\Sigma(\mathbf{k}, E)=G_{0}^{-1}(\mathbf{k}, E)-G^{-1}(\mathbf{k}, E){ }^{26,27}$ Figure 1 is the energy-dependence of the calculated real [Fig. 1(a)] and imaginary [Fig. 1(b)] parts of the self-energy function for $n_{\text {imp }}=0.1 \%$. Within the energy range of $[-0.02 t, 0.02 t]$ (depending on the vacancy concentration), both $\operatorname{Re} \Sigma$ and $\operatorname{Im} \Sigma$ depend explicitly on the momentum, as shown in Figs. 1(c) and 1(d). This momentum dependence of the self-energy causes the failure of the ATA or SCTA. ${ }^{28}$ It also shows that the effective homogeneous medium approximation is invalid around the Dirac point. Beyond this energy range, however, our simulations show insensitiveness of the self-energy function to wave vector $\mathbf{k}$, and $\Sigma$ varies only with the energy. More interestingly, a spike in $\operatorname{Re} \Sigma$ around the Dirac point is observed in Fig. 1(c), in contrast with the ATA and SCTA. ${ }^{17,20}$ Another notable feature is that the ATA predicts a peak of $\operatorname{Im} \Sigma$ at the Dirac point. Instead, a dip at the Dirac point [Fig. 1(d)] is observed. Though the self-energy function shows a complicated momentum and energy dependence around the Dirac point, we find that it still satisfies the Kramers-Kronig relation. ${ }^{27}$ In order to have a better picture of the quasiparticles of the system, we compute the spectral function below.

The single-particle spectral function relates to the Green's function through $A(\mathbf{k} \pm, E)=-\operatorname{Im} G(\mathbf{k} \pm, E) / \pi \cdot{ }^{27}$ Generally speaking, the spectral function $A_{0}(\mathbf{k}, E)$ is a $\delta$ function in the absence of disorders, reflecting that the wave vector $\mathbf{k}$ is a good quantum number and all its weight ratio is precisely at energy $E=E_{k \pm}$. In the presence of disorders, the $\delta$ peak is broadened due to finite lifetime of quasiparticles, resulting from the disorder-scattering effect. The linewidth of the peak is given by $\operatorname{Im} \Sigma(E)$ that measures the elastic relaxation lifetime
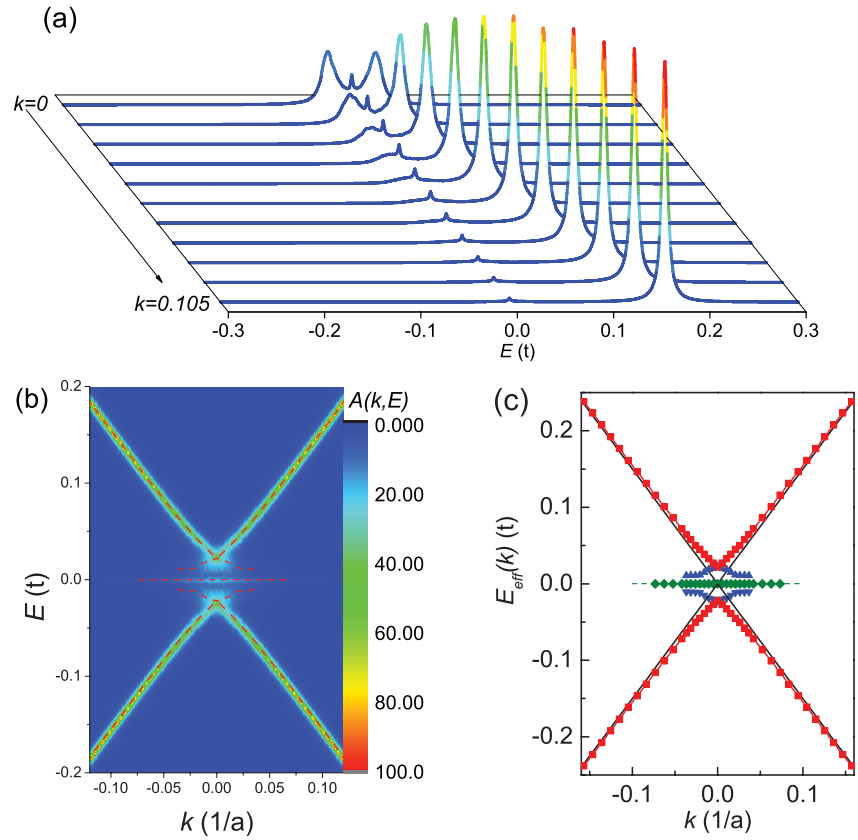

FIG. 2. (Color online) (a) Quasiparticle spectral function $A(\mathbf{k}+, E)$ plotted as a function of energy $E$ for various wave vectors at vacancy concentration $n_{\mathrm{imp}}=0.1 \% . A(\mathbf{k}-, E)$ can be obtained by reflecting $A(\mathbf{k}+, E)$ around $E=0$ due to the particle-hole symmetry. (b) Density plot of spectral function $A(\mathbf{k}, E)=A(\mathbf{k}+, E)+$ $A(\mathbf{k}-, E)$ in $\mathbf{k}-E$ plane for $n_{\mathrm{imp}}=0.1 \%$. (c) Dispersion relation (dotted line) extracted from (b). The blue triangles and green diamonds are the new resonant band and dispersionless zero-energy band, respectively. For comparison, the linear dispersion of clean graphene is plotted as the black solid lines.

$\tau_{e}, \tau_{e}=\frac{\hbar}{-2 \operatorname{Im} \Sigma(E)}$. These general features are indeed observed in the weak point potential scattering cases. ${ }^{16}$ The spectral function is qualitatively different in the strongly resonant scattering regime. Taking $A(k=0+, E)$ in Fig. 2(a) as an example, the spectral function is surprisingly split into three peaks: one broadened peak $\left(p_{-}\right)$centering in the hole regime $(E<0)$, one broadened peak $\left(p_{+}\right)$centering in the electron regime $(E>0)$, and one sharp peak $\left(p_{0}\right)$ at charge neutrality point $(E=0)$. The $p_{-}$peak moves toward $E=0$ as the wave vector $\mathbf{k}$ increases, while its height is reduced and its width increases. $p_{0}$ peak position do not change with $\mathbf{k}$ while its height decreases with $\mathbf{k}$. Meanwhile, the $p_{+}$peak moves away from $E=0$ and its height is significantly increased and its width is narrowed. When the wave vector exceeds a threshold value, the $p_{-}$and the $p_{0}$ peaks disappear.

By tracing the trajectories of the peaks of the density plot of spectral function $A(\mathbf{k}, E)=A(\mathbf{k}+, E)+A(\mathbf{k}-, E)$ in the $k-E$ plane, shown in Fig. 2(b), one can obtain the dispersion relation $E(\mathbf{k})$ shown in Fig. 2(c). Many other physical quantities like the group velocity and elastic scattering time can be directly extracted from Figs. 2(b) and 2(c). ${ }^{16}$ Away from the Dirac point, the $p_{+}$peak dominates the spectral function and the effective energy dispersion approaches the linear behavior (red square dotted line). Around the Dirac point, Fig. 2(b) clearly shows the anomalous band structure of quasiparticle. Importantly, an exotic dispersion (blue triangles dotted line) with finite lifetime is observed, corresponding 
to the $p_{-}$peak in the spectral function. This indicates that a split of host band of pristine graphene happens near the Dirac point. A dispersionless band (green diamond dotted line) develops at zero-energy, corresponding to the $p_{0}$ peak in the spectral function relating to the kink in $\operatorname{Re} \Sigma$ and the dip in $\operatorname{Im} \Sigma$. When the electron-electron interaction is switched on, this zero-bandwidth dispersionless band may lead to ferromagnetic or antiferromagnetic ground state for the system. ${ }^{29-31}$ In experiments, the direct evidence of the corresponding split of the Dirac point and the dispersionless band has indeed been reported in hydrogenated graphene by ARPES. ${ }^{7,8}$

To understand the physical origin of above results, let us start with the exact result for one vacancy. ${ }^{32}$ A vacancy deducts one level from the continue band and at the same time pulls out another level from the continue band to a localized zero energy single-vacancy state due to the particle-hole symmetry. ${ }^{33,34}$ For two vacancies on different sublattices, two single-vacancy states split into bonding and antibonding pairs through hybridization. ${ }^{30}$ The energy split decreases exponentially with the vacancy-vacancy distance. For system containing many randomly distributed vacancies (with equal number of vacancies on different sublattices), the situation is complicated. According to Ref. 34, there is no zero-energy eigenfunction that vanishes on one sublattice in this case. However, we indeed observe zero-energy states forming dispersionless bands within our numerical accuracy, in a direct conflict with the above claim. ${ }^{34}$ The zero-energy states observed here may arise from the quantum interference effect. Through the coherent multiple scattering, some singlevacancy states are strongly coupled so that their energy split off away from zero energy, leading to the split of spectrum. Due to the destructive quantum interference, some of the single-vacancy states are effectively decoupled from other vacancy states and thus form the zero energy states. Therefore, the zero-energy states and split of spectrum appear. In order to confirm the above viewpoint, we look at vacancy concentration dependence of the energy split. Figure 3(a) is the spectral function at wave vector $\mathbf{k}=0$. One striking feature is the energy split $\Delta E$ (peak-to-peak distance between $p_{+}$and $p_{-}$
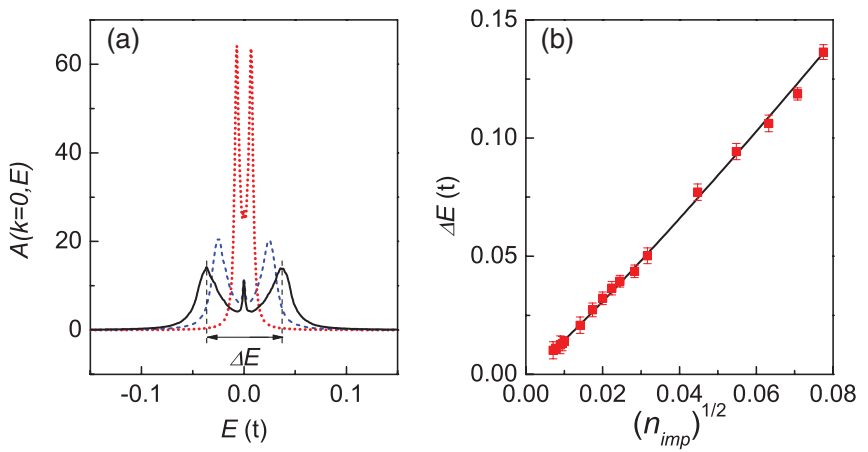

FIG. 3. (Color online) (a) Quasiparticle spectral function $A(\mathbf{k}=$ $0, E)$ as a function of energy $E$ for several vacancy concentrations: $n_{\text {imp }}=0.01 \%$ (red dotted line), $n_{\text {imp }}=0.1 \%$ (blue dashed line), and $n_{\text {imp }}=0.2 \%$ (black solid line). $\Delta E$ is the energy split between the two resonant peaks. (b) The vacancy concentration dependence of the energy split $\Delta E$ (red dots). The $x$ axis is in $\left(n_{\text {imp }}\right)^{1 / 2}$. The black line is the linear fit $\Delta E=A\left(n_{\text {imp }}\right)^{1 / 2}$ with $A=1.76$. peaks). $\Delta E$ is the energy split between two nodal points at $\mathbf{k}=$ 0 (neglecting the zero-energy band) in Fig. 2(c). The vacancy concentration dependence of $\Delta E$ is plotted in Fig. 3(b), where the $x$ axis is the square root of $n_{\text {imp }}$ that measures the inverse of vacancy-to-vacancy distance $L_{v} \sim 1 / \sqrt{n_{\text {imp }}}$. The square-root dependence of $\Delta E \propto \sqrt{n_{\mathrm{imp}}} \propto 1 / L_{v}$ supports the picture that the coherent scattering among the vacancies play an important role, which is significantly different from the case for two vacancies. ${ }^{30}$ It can also explain that the splitting behavior is remarkable around the Dirac point where the electronic de Broglie wavelength $\lambda$ is much bigger than $L_{v}$. As the wave vector exceeds a threshold so that the wavelength is small $\lambda \ll L_{v}$, the quasiparticle is insensitive to the coupling of resonant states. Hence, the new dispersive band disappears for the short wavelength. This is why ATA approximate approach works well far from the Dirac point. Of course, a further careful investigation is needed in order to find the true origin of the zero-energy states and to have a deeper understanding of quantum interference effect on energy levels.

To make our results even more convincing, we revisit the calculation of the DOS based on our spectral function $\rho(E)=\frac{1}{N} \sum_{k} A(k, E)$. As shown in Fig. 4(a), a sharp peak developed at $E=0$ and energy regime influenced by vacancies is approximately proportional to $\Delta E \sim \sqrt{n_{\mathrm{imp}}}$. All of these results are consistent with previous numerical simulations. ${ }^{14,15}$ This implies that the anomalous DOS comes from the formation of the new band structure near the Dirac point. It also proves that our spectral analysis captures the correct physics near the Dirac point, which is failed by the ATA or SCTA. ${ }^{17}$

Figure 4(b) is the calculated conductivity from the Kubo formula at the zero-temperature $\sigma_{x x}=$ $\frac{\hbar}{2 \pi L^{2}} \overline{\operatorname{Tr}\left[j_{x} G^{R}(E) j_{x} G^{A}(E)\right]}$, within the semiclassical Drude-Boltzmann approximation $\overline{G^{R} G^{A}} \simeq \overline{G^{R}} \overline{G^{A}} \cdot{ }^{28} \mathrm{~A}$ conductivity plateau of $4 e^{2} / \pi h$ is observed near the charge neutrality point. Taking into account the weak localization correction, the conductivity near the Dirac point may become lower than the semiclassical value. ${ }^{35}$ Interestingly, the plateau width increases with the vacancy concentration. ${ }^{13,36-38}$ The plateau width in terms of energy $\Delta E_{c}$ is almost
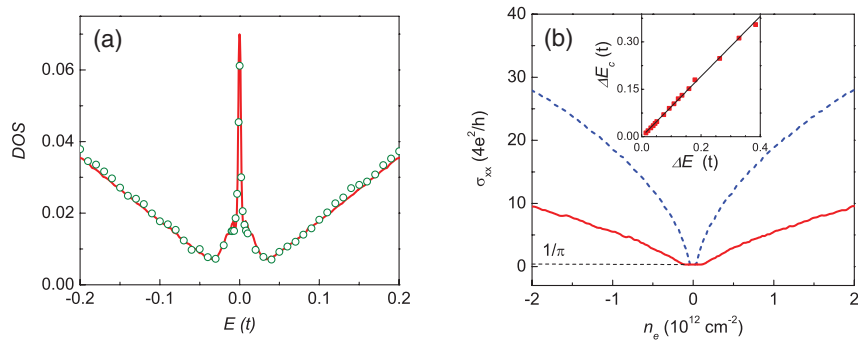

FIG. 4. (Color online) (a) Comparison of the DOS obtained from our spectral function (red line) with the numerical simulation of Ref. 15 (green dots) for $n_{\mathrm{imp}}=0.1 \%$. Excellent agreement demonstrates the accuracy of our calculations. (b) Conductivity as a function of charge density $n_{e}$ for $n_{\text {imp }}=0.01 \%$ (blue dashed line) and $n_{\text {imp }}=0.1 \%$ (red solid line), respectively. Inset: The conductivity plateau width in terms of energy $\Delta E_{c}$ vs. energy split $\Delta E$ shown in Fig. 3. $\Delta E_{c}$ can be obtained from the relationship $n_{e}=\int{ }^{E_{F}} \rho(E) d E$. Black line is the linear fit of $\Delta E_{c}=A \Delta E$ with $A=0.95$. 
equal to the energy split $\Delta E$ as shown in the inset of Fig. 4(b), which implies that the origin of the plateau is the anomalous band structure near the Dirac point. This conductivity plateau provides an alternative explanation to the experimental observation. ${ }^{39}$ It should be pointed out that our calculations do not include the contribution from the impurity-band-induced variable range-hopping conduction, which may also be important in understanding the experiments at finite temperatures. ${ }^{8,10}$

In conclusion, we have studied vacancy-induced resonantscattering effects on the one-electron properties of graphene. From our accurate spectral function, a new quasiparticle dispersion band due to the coherent scattering between neighbor

*wliustc@mail.ustc.edu.cn

†phxwan@ust.hk

${ }^{1}$ K. S. Novoselov, A. K. Geim, S. V. Morozov, D. Jiang, Y. Zhang, S. V. Dubonos, I. V. Grigorieva, and A. A. Firsov, Science 306, 666 (2004).

${ }^{2}$ A. K. Geim and K. S. Novoselov, Nat. Mater. 6, 183 (2007).

${ }^{3}$ K. Nomura and A. H. MacDonald, Phys. Rev. Lett. 98, 076602 (2007).

${ }^{4}$ Z. Y. Liu, X. X. Zhang, Y. W. Mao, Y. Y. Zhu, Z. Y. Yang, C. T. Chan, and P. Sheng, Science 289, 1734 (2000).

${ }^{5}$ T. W. Ebbesen, H. J. Lezec, H. F. Ghaemi, T. Thio, and P. A. Wolff, Nature(London) 391, 667 (1998).

${ }^{6}$ L. Martin-Moreno, F. J. Garcia-Vidal, H. J. Lezec, K. M. Pellerin, T. Thio, J. B. Pendry, and T. W. Ebbesen, Phys. Rev. Lett. 86, 1114 (2001).

${ }^{7}$ D. Haberer, D. V. Vyalikh, S. Taioli, B. Dora, M. Farjam, J. Fink, D. Marchenko, T. Pichler, K. Ziegler, S. Simonucci, M. S. Dresselhaus, M. Knupfer, B. Buchner, and A. Gruneis, Nano Lett. 10, 3360 (2010).

${ }^{8}$ D. Haberer, L. Petaccia, M. Farjam, S. Taioli, S. A. Jafari, A. Nefedov, W. Zhang, L. Calliari, G. Scarduelli, B. Dora, D. V. Vyalikh, T. Pichler, C. Woll, D. Alfe, S. Simonucci, M. S. Dresselhaus, M. Knupfer, B. Buchner, and A. Gruneis, Phys. Rev. B 83, 165433 (2011).

${ }^{9}$ A. Bostwick, J. L. McChesney, K. V. Emtsev, T. Seyller, K. Horn, S. D. Kevan, and E. Rotenberg, Phys. Rev. Lett. 103, 056404 (2009).

${ }^{10}$ J. H. Chen, W. G. Cullen, C. Jang, M. S. Fuhrer, and E. D. Williams, Phys. Rev. Lett. 102, 236805 (2009).

${ }^{11}$ T. Stauber, N. M. R. Peres, and F. Guinea, Phys. Rev. B 76, 205423 (2007).

${ }^{12}$ J. P. Robinson, H. Schomerus, L. Oroszlany, and V. I. Fal'ko, Phys. Rev. Lett. 101, 196803 (2008).

${ }^{13}$ T. O. Wehling, S. Yuan, A. I. Lichtenstein, A. K. Geim, and M. I. Katsnelson, Phys. Rev. Lett. 105, 056802 (2010).

${ }^{14}$ V. M. Pereira, J. M. B. LopesdosSantos, and A. H. CastroNeto, Phys. Rev. B 77, 115109 (2008).

${ }^{15}$ S. D. Wu, L. Jing, Q. X. Li, Q. W. Shi, J. Chen, H. B. Su, X. P. Wang, and J. L. Yang, Phys. Rev. B 77, 195411 (2008).

${ }^{16}$ W. Zhu, Q. W. Shi, X. R. Wang, X. P. Wang, J. L. Yang, J. Chen, and J. G. Hou, Phys. Rev. B 82, 153405 (2010)

${ }^{17}$ N. M. R. Peres, F. Guinea, and A. H. CastroNeto, Phys. Rev. B 73, 125411 (2006). vacancies is predicted, and each Dirac point splits into two new nodal points. Furthermore, a dispersionless band is developed at zero energy. This result sheds light on the characteristics of electronic structures and transport properties of graphene in the presence of resonant impurities.

We thank the referees for valuable suggestions. This work is partially supported by NNSF of China (No. 10974187, No. 10874165), by NKBRP of China (No. 2011CB921403, No. 2012CB922003), and by KIP of the CAS (No. KJCX2YW-W22). X.R.W. acknowledges the support of Hong Kong RGC (Grant No. 604109, No. RPC11SC05, and No. HKUST17/CRF/08).

${ }^{18}$ S. S. Pershoguba, Y. V. Skrypnyk, and V. M. Loktev, Phys. Rev. B 80, 214201 (2009); Y. V. Skrypnyk and V. M. Loktev, ibid. 83, 085421 (2011); e-print arXiv:1105.4907.

${ }^{19}$ M. Farjam, D. Haberer, and A. Gruneis, Phys. Rev. B 83, 193411 (2011).

${ }^{20}$ T. Lofwander and M. Fogelstrom, Phys. Rev. B 76, 193401 (2007).

${ }^{21}$ S. Ihnatsenka and G. Kirczenow, Phys. Rev. B 83, 245442 (2011).

${ }^{22}$ Our calculations show that the small imbalance of vacancies on two sublattices does not change our results.

${ }^{23}$ J. K. Cullum and R. A. Willoughby, Lancozs Algorithms for Large Symmetric Eigenvalue Problem (Birkhäuser, Boston, 1985).

${ }^{24}$ E. Dagotto, Rev. Mod. Phys. 66, 763 (1994); L. C. Davis, Phys. Rev. B 28, 6961 (1983); B. Bauml, G. Wellein, and H. Fehske, ibid. 58, 3663 (1998); S. M. Anlage and D. L. Smith, ibid.34, 2336 (1986).

${ }^{25}$ L. W. Wang, L. Bellaiche, S. H. Wei, and A. Zunger, Phys. Rev. Lett. 80, 4725 (1998); Y. Zhang, A. Mascarenhas, and L. W. Wang, ibid. 101, 036403 (2008).

${ }^{26}$ E. N. Economou, Green's Functions in Quantum Physics (Springer, New York, 2006).

${ }^{27}$ A. Altland and B. Simons, Condensed Matter Field Theory (Cambridge University Press, Cambridge, 2006).

${ }^{28}$ E. Akkermans and G. Montambaux, Mesoscopic Physics of Electrons and Photons (Cambridge University Press, Cambridge, 2007).

${ }^{29}$ Elliott H. Lieb, Phys. Rev. Lett. 62, 1201 (1989).

${ }^{30}$ J. J. Palacios, J. Fernandez-Rossier, and L. Brey, Phys. Rev. B 77, 195428 (2008)

${ }^{31}$ D. Soriano, N. Leconte, P. Ordejon, J. C. Charlier, J. J. Palacios, and S. Roche, Phys. Rev. Lett. 107, 016602 (2011).

${ }^{32}$ V. M. Pereira, F. Guinea, J. M. B. LopesdosSantos, N. M. R. Peres, and A. H. CastroNeto, Phys. Rev. Lett. 96, 036801 (2006).

${ }^{33} \mathrm{~K}$. Yang (Private communication).

${ }^{34}$ M. Inui, S. A. Trugman, and E. Abrahams, Phys. Rev. B 49, 3190 (1994).

${ }^{35}$ N. Leconte, D. Soriano, S. Roche, P. Ordejon, J.-C. Charlier, and J. J. Palacios, Acs Nano 5, 3987 (2011).

${ }^{36}$ S. J. Yuan, H. De Raedt, and M. I. Katsnelson, Phys. Rev. B 82, 115448 (2010)

${ }^{37}$ A. Ferreira, J. Viana-Gomes, J. Nilsson, E. R. Mucciolo, N. M. R. Peres, and A. H. CastroNeto, Phys. Rev. B 83, 165402 (2011).

${ }^{38}$ A. Lherbier, Simon M.-M. Dubois, X. Declerck, S. Roche, Y. M. Niquet, and J. C. Charlier, Phys. Rev. Lett. 106, 046803 (2011).

${ }^{39}$ Z. H. Ni, L. A. Ponomarenko, R. R. Nair, R. Yang, S. Anissimova, I. V. Grigorieva, F. Schedin, P. Blake, Z. X. Shen, E. H. Hill, K. S. Novoselov, and A. K. Geim, Nano. Lett. 10, 3868 (2010). 